\title{
Prevalence of vitreomacular adhesion: an optical coherence tomography analysis in the retina clinic setting
}

This article was published in the following Dove Press journal:

Clinical Ophthalmology

6 April 2016

Number of times this article has been viewed

\section{Elias Reichel' \\ Glenn J Jaffe ${ }^{2}$ \\ Srinivas R Sadda ${ }^{3}$ \\ Stefanie Schuman ${ }^{2}$ \\ Amir $\mathrm{H} \mathrm{Hariri}{ }^{3}$ \\ Keegan Skidmore' \\ Jake Duker'}

'Department of Ophthalmology, New England Eye Center, Tufts

Medical Center, Tufts University School of Medicine, Boston, MA, ${ }^{2}$ Duke Eye Center, Duke Reading Center, Durham, NC ${ }^{3}$ Department of Ophthalmology, Doheny Eye Centers, University of California, Los Angeles, CA, USA
Correspondence: Elias Reichel Department of Ophthalmology, New England Eye Center, Tufts Medical Center, Tufts University School of Medicine, 800 Washington Street, Box 450, Boston, MA 021II, USA

$\mathrm{Tel}+\mathrm{I} 6176361648$

Fax +I 6176364866

Email ereichel@tuftsmedicalcenter.org
Purpose: The aims of this study were to determine the prevalence of vitreomacular adhesion (VMA) in a random sample of clinical patients at three US retina clinics and to assess comorbid retinal conditions, ocular diseases, prior treatment history, and other medical histories.

Patients and methods: This observational, retrospective cohort study was based on patients from the Doheny Eye Centers, Duke Eye Center, and Tufts Medical Center who received a bilateral spectral domain optical coherence tomography (SD-OCT) scan (one scan/eye) for clinical evaluation with available medical records. The study had three phases: 1) collection of retrospective patient data; 2) review of OCT scans at a reading center to assess VMA and associated conditions; and 3 ) analyses and reporting of data on the prevalence of VMA, patient demographics, and comorbid conditions. Data were obtained from electronic health records and OCT grading forms. Outcome measures from bilateral SD-OCT scans and medical records included OCT evaluation of VMA and retinal comorbid conditions.

Results: In 719 patients with 1,483 reviewable OCT scans, the prevalence of VMA was estimated at $14.74 \%(90 \% \mathrm{CI}, 12.58 \%-16.92 \%)$. The prevalence of unilateral VMA was estimated at $12.39 \%$, while bilateral VMA was $2.36 \%$. In patients with VMA, 34 out of 123 eyes with VMA (27.64\%) also had fovea deformed by vitreomacular traction. Macular hole (MH) was significantly more prevalent in VMA-diagnosed eyes versus non-VMA-diagnosed eyes $(6.5 \%$ versus $1.9 \% ; P=0.02)$. There was a significantly higher incidence of full-thickness $\mathrm{MH}$ $(P=0.008)$, operculum/flaps $(P<0.0001)$, and lamellar or pseudo-holes $(P=0.048)$ in VMAdiagnosed versus non-VMA-diagnosed eyes. Age, $\mathrm{MH}$ as a comorbid condition, full-thickness $\mathrm{MH}$, lamellar or pseudo-holes, and operculum were predictive of a VMA diagnosis.

Conclusion: The prevalence of VMA was estimated at $14.74 \%$ in a random sample of patients from three retina clinics. VMA diagnosis can be predicted by factors, including age, $\mathrm{MH}$ as a comorbid condition, and lamellar or pseudo-holes.

Keywords: VMA, OCT, macular hole, vitreomacular traction, comorbid conditions, operculum/ flaps, lamellar or pseudo-holes

\section{Introduction}

Vitreomacular adhesion (VMA) occurs when there is incomplete or anomalous posterior vitreous detachment from the retinal internal limiting membrane. ${ }^{1-4}$ VMA can be asymptomatic without sequelae. In some cases, however, VMA can be symptomatic, contributing to the pathogenesis and clinical course of various ocular conditions, ${ }^{5}$ including vitreomacular traction (VMT) syndrome, ${ }^{6}$ idiopathic macular hole $(\mathrm{MH}),{ }^{7}$ cystoid macular edema, ${ }^{8}$ diabetic macular edema (DME), ${ }^{9}$ and age-related macular degeneration (AMD). ${ }^{10}$ Symptomatic VMA can be associated with loss of visual function. ${ }^{11}$ It has 
been estimated that $1.5 \%$ of the population has eye disease caused by, or associated with, VMA. ${ }^{11}$ There are limited epidemiological data on VMT syndrome, MH, DME, and other conditions linked to VMA, and in certain conditions, the relationship to VMA is not clear. ${ }^{12}$ In a meta-analysis, eyes with neovascular AMD were two times more likely to have VMA compared with controls. In addition, VMT was present in $28.7 \%$ of eyes with DME. ${ }^{12}$ Such estimates are typically based on small sample sizes. ${ }^{12}$ The prevalence of symptomatic VMA is not well documented. With imaging techniques such as optical coherence tomography (OCT), it may be possible to estimate VMA prevalence. OCT, a noninvasive technique used to diagnose macular conditions, has been increasingly used to identify VMA, because it is more sensitive than using clinical examination alone. ${ }^{13,14}$

In the present study, the primary objective was to estimate the prevalence of VMA in a random sample of new patients presenting at three US retina clinics. The secondary objective was to determine the distribution of comorbid retinal conditions (including epiretinal membrane, $\mathrm{MH}, \mathrm{AMD}$, retinal vein occlusion, DME, and diabetic retinopathy), other ocular diseases, prior treatment history, and other medical histories within the study population. Additionally, the proportion of patients with unilateral versus bilateral VMA and the correlation between VMA and visual acuity and other visual outcomes were determined.

\section{Materials and methods}

\section{Study design}

This was an observational, retrospective cohort study consisting of a random sample of patients who received a bilateral spectral domain OCT (SD-OCT) scans (typically one volume scan/eye) at one of the three tertiary retina clinics (Doheny Eye Centers, University of California, Los Angeles, CA, USA; Duke Eye Center, Durham, NC, USA; Tufts Medical Center, Boston, MA, USA) between 2009 and 2012. The study was conducted in three phases: 1) collection of retrospective patient data; 2) review of OCT scans at a reading center to assess VMA and associated conditions; 3 ) analyses and reporting of data on the prevalence of VMA in the study cohort along with patient demographics and other comorbid conditions.

Data for this study were obtained from available patient medical records. Data were also obtained from OCT grading forms, in which information was recorded regarding the readability of the scans and associated ocular conditions. The investigators agreed on study definitions and standards for reading OCT scans and interpreting medical record information prior to the study commencing. Certified readers at a single core facility, the Duke Reading Center, were used to maintain consistent interpretation and diagnosis of VMA. This study was conducted in accordance with International Society for Pharmacoepidemiology (ISPE) Guidelines for Good Epidemiology Practices, and with applicable regulatory requirements. Since the data collection for this study includes only de-identified data to be obtained during a retrospective chart review, informed consent was not required.

\section{Study population}

This study comprised a random sample of $\sim 750$ patients who had received a bilateral SD-OCT scan at one of the three retina clinics. Patients were included in the study if they had presented for clinical evaluation as part of a diagnostic workup or follow-up evaluations (rather than as part of a clinical trial), had bilateral SD-OCT scan data available (regardless of SD-OCT manufacturer or scan density), and had medical records available with sections of the record required for data collection.

Patients were excluded from the study if they had a history of vitrectomy in either eye, had scans performed using time-domain OCT, or if they only had unilateral SD-OCT images available.

As multiple scans had been taken for patients during the course of the study, primary data points were derived from the patient's first visit and the first set of scans. These were used to estimate the point prevalence of VMA per patient, per eye, when the SD-OCT scan image was obtained.

\section{Sample size determination}

Based on discussions among the investigators, the underlying prevalence of VMA was estimated to be $12 \%$ across the three retina clinic sites. It was assumed that $\sim 5 \%$ of patient records would be ungradable. Thus, for a two-sided $90 \%$ confidence interval (CI), with a margin of error of $\pm 2 \%$, 750 patient records were needed to achieve the required sample size of 715 patients.

\section{Study endpoints}

Data for this study were collected in three parts. In the first part, screening information was collected that indicated whether patients met the inclusion criteria. In the second part, data were obtained from certified image readers' evaluations of SD-OCT images for the presence/absence of VMA in each eye and comorbid retinal conditions. In these evaluations, an eye was identified as having VMA if it had any visible vitreous separation and if there was any attachment of the posterior hyaloid within the central 2,000 $\mu \mathrm{m}$ diameter centered on the foveola, which did not extend beyond the $2,000 \mu \mathrm{m}$ diameter in either one or both directions on the 
horizontal line scan $(6 \mathrm{~mm}$ field $=$ macula $=$ cirrus $512 \times 128$ volume scan [Carl Zeiss Meditec AG, Dublin, CA, USA] or Spectralis $20^{\circ} \times 20^{\circ}$ cube [Heidelberg Engineering, Heidelberg, Germany]). The Doheny Eye Centers conducted a minimum of 49 B-scans, Tufts Medical Center conducted 128 B-scans with 512 A-scans per B-scan, while Duke Eye Center used both the 49 cross-sectional B-scan images, each composed of 512 A-scans, and the 128 cross-sectional B-scan images, each composed of $512 \mathrm{~A}$-scans. In the third part, medical record data were collected on patient demographics, including age, sex, race, underlying ophthalmic conditions, treatment history, and medical history.

\section{Data management and analysis}

Data collected from patient charts and medical records were entered into the study database using the Viedoc ${ }^{\mathrm{TM}}$ electronic data capture system (ACI Clinical, Bala Cynwyd, PA, USA). Data analyses were conducted using SAS version 9.3 (SAS Institute Inc., Cary, NC, USA) and NCSS 8 NCSS (LLC, Kaysville, UT, USA). A two-sided 90\% CI was constructed to calculate the prevalence of VMA. For continuous variables, mean \pm standard deviation (SD), median, minimum, and maximum values were calculated and Student's $t$-test was used to compare individuals with and without a VMA diagnosis. Categorical variable counts and percentages were determined and the chi-square test for homogeneity was used to compare the two VMA diagnostic groups. A two-tailed $P$-value of $<0.05$ was used to determine statistical significance. Results were reported as a descriptive analysis including summary statistics and/or frequency distributions as appropriate.

\section{Results}

\section{Patient numbers and data site distribution}

This study was conducted between 2009 and 2012 during which a total of 790 patients, 1,575 SD-OCT scans, and 823 medical records were collected from the three study sites. Seventy-one $(9.0 \%)$ patients were excluded because of various reasons: the main reasons were medical records with no OCT scans or OCT scans that were ungradable. A total of 719 patients were included in the final sample size, each having a separate scan per eye. Therefore, there were 1,438 reviewable SD-OCT scans. A similar number of patients were studied at each of the three sites (Figure 1).

\section{Patient baseline characteristics}

Of the 719 patients included in the study, $45 \%$ were males and the mean (SD) age was 59 (18.43) years. Participants were white (28\%), black (6\%), and Asian (2\%); however, data regarding race were not available for the majority $(60 \%)$ of patients, and no significant differences were observed $(P=0.25)$. Patients with VMA were significantly older compared with those without VMA (66 years versus 58 years, $P<0.0001$ ), and there was no difference in the percentage of males with or without VMA $(P=0.97$; Table 1).

\section{Prevalence of VMA}

The prevalence of VMA was similar at each of the study sites (Table 1). Of the 719 patients, 106 were diagnosed with VMA: 89 of whom had unilateral VMA and 17 had bilateral VMA (Figure 2). The prevalence of VMA was estimated at $14.74 \%$ (90\% CI, 12.58\%-16.92\%). The estimated prevalence of unilateral VMA was $12.39 \%$ and bilateral VMA was $2.36 \%$. Additionally, 34 out of 123 eyes with VMA (27.64\%) also had fovea deformed by VMT. The remaining 18 eyes $(2.37 \%)$ were ungradable.

\section{Comorbid retinal conditions from medical record data}

The distribution and number of comorbid retinal conditions were assessed from medical record data. $\mathrm{MH}$ was significantly more prevalent in VMA-diagnosed eyes versus

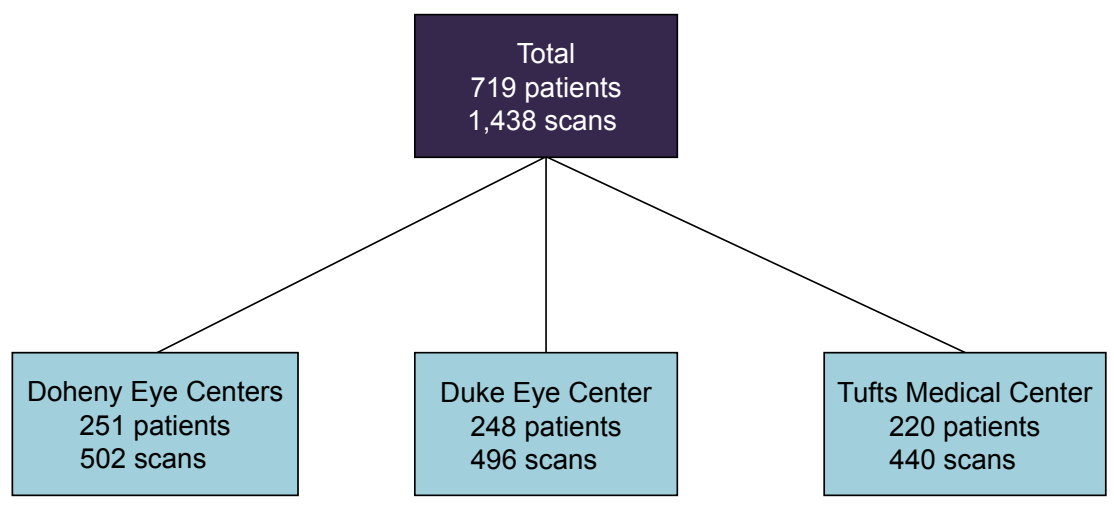

Figure I Flowchart for patients $(\mathrm{N}=719)$ and data site distribution. 
Table I Patient baseline demographics and VMA diagnosis

\begin{tabular}{|c|c|c|c|c|}
\hline \multirow[b]{2}{*}{ Demographic } & \multirow[b]{2}{*}{ All patients } & \multicolumn{2}{|l|}{ VMA diagnosis } & \multirow[b]{2}{*}{$P$-value } \\
\hline & & $\begin{array}{l}\text { Patients with VMA diagnosis } \\
\text { on OCT scan (either eye) }\end{array}$ & $\begin{array}{l}\text { Patients with no VMA } \\
\text { diagnosis (both eyes) }\end{array}$ & \\
\hline Number of patients & $719^{a}$ & 106 & 613 & \\
\hline Distribution by center, n (\%) & & & & $0.67^{\mathrm{b}}$ \\
\hline Doheny Eye Centers & 251 & $33(3|| 3)$. & $218(35.56)$ & \\
\hline Duke Eye Center & 248 & 39 (36.79) & 209 (34.09) & \\
\hline Tufts Medical Center & 220 & $34(32.08)$ & $186(30.34)$ & \\
\hline Sex & & & & $0.97^{\mathrm{b}}$ \\
\hline Males, n (\%) & $320(44.5 I)$ & $47(44.34)$ & $273(44.54)$ & \\
\hline Race, n (\%) & & & & $0.25^{\mathrm{b}}$ \\
\hline White & $203(28.23)$ & $36(33.96)$ & $167(27.24)$ & \\
\hline Black & $43(5.98)$ & $6(5.66)$ & $37(6.04)$ & \\
\hline Asian & $17(2.36)$ & I (0.94) & $16(2.6 \mathrm{I})$ & \\
\hline Other & $22(3.06)$ & I (0.94) & $21(3.43)$ & \\
\hline Not available ${ }^{c}$ & $434(60.36)$ & $62(58.49)$ & $372(60.69)$ & \\
\hline Number of patients & $718^{d}$ & 106 & 612 & \\
\hline \multicolumn{5}{|l|}{ Age, years } \\
\hline Mean (SD) & $58.84(18.43)$ & $65.76(13.01)$ & $57.65(18.96)$ & $<0.0001^{\mathrm{e}}$ \\
\hline Median (range) & $62(7-98)$ & $68(25-9 \mid)$ & 61 (7-98) & \\
\hline
\end{tabular}

Notes: ${ }^{\mathrm{a} T o t a l}$ number of patients included in the study. ${ }^{\mathrm{b}} \mathrm{Chi}$-square test. ${ }^{\mathrm{c}}$ Most responses were not available for Tufts Medical Center ( $99.55 \%$ of patients) and Doheny Eye

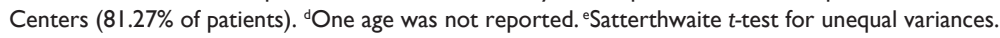

Abbreviations: OCT, optical coherence tomography, VMA, vitreomacular adhesion; SD, standard deviation.

non-VMA-diagnosed eyes $(6.5 \%$ versus $1.9 \% ; P=0.02)$. There were no significant differences in any of the other retinal comorbidities between the two cohorts. In addition, comorbid conditions occurred in higher numbers in patients diagnosed with VMA compared with patients not diagnosed with VMA (Table 2). Two patients were reported as having VMT but were included in the "no VMA diagnosis" group. In these cases, the VMT diagnosis was obtained from medical record data and analyzed against the Duke Reading Center's definition of VMA. Therefore, the patients may have had VMT at some point, but the reading center assessment of the OCT scan did not indicate a VMA diagnosis.

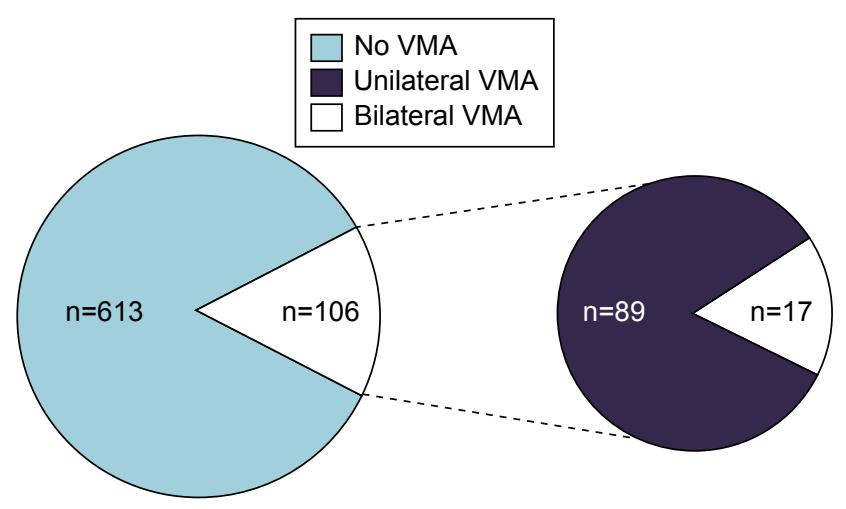

Figure 2 Proportion of patients with different types of VMA. Abbreviation: VMA, vitreomacular adhesion.
No other evaluated variables were found to be significantly associated with VMA diagnosis, including the occurrence of asthma, coronary artery disease, diabetes, hypertension, hyperlipidemia, and hypothyroidism. Furthermore, prior ocular interventions such as retina laser surgery, capsule surgery, cataract surgery, and prior intravitreal injections were not shown to be predictive of VMA diagnosis in this patient population (data not shown).

\section{OCT grading form data}

Based on the analysis of the data from the Duke Reading Center's OCT grading forms, there was a significantly higher incidence of full-thickness $\mathrm{MH}(P=0.008)$, operculum/flaps $(P<0.0001)$, and lamellar or pseudo-holes $(P=0.048)$ in eyes with VMA diagnosis versus those with no VMA diagnosis (Table 3). The occurrence of epiretinal membrane, intraretinal cysts, subretinal fluid, photoreceptor disruption, retinal schisis at the fovea, retinal pigment epithelium elevation and/or subretinal hyperreflective material at the foveal center, external limiting membrane intact in the central $1 \mathrm{~mm}$ cube, and inner segment/ outer segment (IS/OS) band intact in the central $1 \mathrm{~mm}$ cube were not significantly predictive of VMA (data not shown).

\section{Discussion}

As people age, the process of posterior vitreous detachment occurs naturally; the vitreous liquefies and releases 
Table 2 Comorbid retinal conditions from medical record data

\begin{tabular}{|c|c|c|c|}
\hline \multirow[b]{2}{*}{ Comorbid retinal condition ${ }^{a}$} & \multicolumn{2}{|l|}{ VMA diagnosis } & \multirow[b]{2}{*}{$P$-value (NS) ${ }^{c}$} \\
\hline & $\begin{array}{l}\text { Eyes with condition } \\
\text { and VMA diagnosis } \\
\mathrm{n}=92(\%)^{\mathrm{b}}\end{array}$ & $\begin{array}{l}\text { Eyes with condition } \\
\text { and no VMA diagnosis } \\
n=534(\%)^{b}\end{array}$ & \\
\hline Diabetic retinopathy & $12(13.04)$ & $49(9.18)$ & All $P$-values $>0.05$ \\
\hline Age-related macular degeneration & $24(26.09)$ & $94(17.6)$ & (except $\mathrm{MH}[P=0.02]$ ) \\
\hline Occlusions (BRVO, CRVO) & $8(8.7)$ & $26(4.87)$ & \\
\hline Uveitis & $5(5.43)$ & $28(5.24)$ & \\
\hline Retinal detachment/posterior & $23(25.0)$ & $99(18.54)$ & \\
\hline \multicolumn{4}{|l|}{ vitreous detachment } \\
\hline Macular hole & $6(6.52)$ & $10(1.87)$ & \\
\hline Epiretinal membrane & $15(16.3)$ & $83(15.54)$ & \\
\hline Vitreous hemorrhage & $2(2.17)$ & $6(1.12)$ & \\
\hline Cataract & $48(52.17)$ & $239(44.76)$ & \\
\hline Trauma & I (I.09) & $2(0.37)$ & \\
\hline Drusen & $5(5.43)$ & $23(4.31)$ & \\
\hline Panretinal photocoagulation & $0(0.0)$ & $4(0.75)$ & \\
\hline Vitreous macular traction & $2(2.17)$ & $2(0.37)^{d}$ & \\
\hline Other & $45(48.91)$ & $308(57.68)$ & \\
\hline $\begin{array}{l}\text { Number of comorbid retinal } \\
\text { conditions }\end{array}$ & $\begin{array}{l}\text { Eyes with condition } \\
\text { and VMA diagnosis } \\
(n=106)\end{array}$ & $\begin{array}{l}\text { Eyes with condition } \\
\text { and no VMA diagnosis } \\
(n=6 \mid 3)\end{array}$ & $P$-value (NS) ${ }^{c}$ \\
\hline 0 & $14(13.21)$ & $79(12.89)$ & All $P$-values $=0.04^{\mathrm{e}}$ \\
\hline I & $31(29.25)$ & $238(38.83)$ & \\
\hline 2 & $33(31.13)$ & $187(30.5 I)$ & \\
\hline 3 & $15(14.15)$ & $80(13.05)$ & \\
\hline 4 & $12(11.32)$ & $25(4.08)$ & \\
\hline 5 & $0(0.00)$ & $3(0.49)$ & \\
\hline 6 & I (0.94) & I (0.0I6) & \\
\hline
\end{tabular}

itself from the vitreoretinal interface..$^{2-4}$ Typically, this is a normal process; however, in some cases, anomalous posterior vitreous detachment can result in VMA. ${ }^{2,15}$ VMA can be asymptomatic, although in some cases it can contribute to various ocular conditions. ${ }^{12}$ When abnormal VMA causes loss of vision, it is referred to as symptomatic VMA. ${ }^{11}$ There are limited epidemiological data available on the prevalence of VMA; however, imaging techniques such as OCT have increased the recognition of VMA and its progressive sight-threatening complications including $\mathrm{MH}{ }^{3}$ In addition, there is now an OCT-based classification system for diseases of the vitreomacular interface as developed by the International Vitreomacular Traction Study Group. The panel has provided characteristic attributes of the clinical stages of VMA, VMT, and MH, as well as recommendations for classification. ${ }^{16}$

The availability of intravitreal ocriplasmin pharmacotherapy as a treatment to relieve $\mathrm{VMA}^{17-20}$ has renewed the

Table 3 OCT grading form data

\begin{tabular}{|c|c|c|c|}
\hline \multirow[t]{3}{*}{ OCT finding } & \multicolumn{2}{|l|}{ VMA diagnosis } & \multirow[t]{3}{*}{$P$-value ${ }^{a}$} \\
\hline & Eyes with condition and & Eyes with condition and no & \\
\hline & VMA diagnosis $(n=\mid 23)$ & VMA diagnosis $(n=I, 3 \mid 5)$ & \\
\hline Full-thickness macular hole & $4.1 \%$ & $0.8 \%$ & 0.008 \\
\hline Operculum/flap & $4.1 \%$ & $0.2 \%$ & $<0.0001$ \\
\hline Lamellar or pseudo-hole & $4.9 \%$ & $1.8 \%$ & 0.048 \\
\hline
\end{tabular}

Note: a Chi-square test (by Monte Carlo simulation of the exact $P$-value).

Abbreviations: OCT, optical coherence tomography; VMA, vitreomacular adhesion. 
interest in VMA and VMT. Ocriplasmin is a proteolytic enzyme indicated for the treatment of symptomatic VMA. ${ }^{21}$ In the present study, we used the International Vitreomacular Traction Study Group Classification, in which VMA and VMT are characterized. ${ }^{16} \mathrm{We}$ did not specifically refer to "symptomatic VMA." The underlying assumption, however, is that most patients with symptomatic VMA would also have VMT.

The primary objective of this observational, retrospective cohort study was to estimate the prevalence of VMA in a random sample of new clinical patients presenting at three retina clinics. The rationale for assessing the prevalence of VMT is that it may provide an estimate of how many patients are presenting to retina clinics who might potentially benefit from the treatment of VMA.

In the 719 patients assessed, the prevalence of VMA, as determined on SD-OCT images, was estimated at $14.74 \%$ (90\% CI, 12.58\%-16.92\%). The prevalence of unilateral VMA was estimated at $12.39 \%$ and as expected was higher than bilateral VMA $(2.36 \%)$. These estimates were based on data collected as part of the clinical evaluation of patients visiting a retina clinic. Other findings showed that further defining VMA, alongside OCT imaging, provided further information on the presence and distribution of comorbid retinal conditions.

With regard to comorbid retinal conditions in VMA, $\mathrm{MH}$ (full-thickness $\mathrm{MH}$, operculum/flaps, or lamellar or pseudo-holes) was significantly more prevalent in eyes with VMA diagnosis compared with those without VMA diagnosis. In addition, age was predictive of a VMA diagnosis.

\section{Conclusion}

The prevalence of VMA was estimated at $14.74 \%$ in a random sample of patients from the three retina clinics in the USA. The diagnosis of VMA can be predicted by factors, including age, $\mathrm{MH}$ as a comorbid condition, and lamellar or pseudo-holes. Although data collected in this study may not be representative of a general population, ${ }^{22}$ the findings presented here provide an indication of the percentage of patients seen in tertiary care retina clinics who have VMA, as determined by OCT. These results may contribute to the identification of patients who might require treatment and management of their VMA to prevent further deterioration. Further investigation into the prevalence of VMA is required. To this end, a Phase I observational study is currently investigating the prevalence of VMA in patients aged $\geq 40$ years (NCT02160340).

\section{Acknowledgments}

The authors would like to thank Andrew Layton of Quorum Consulting, Inc. for his contribution to the study, specifically the execution of the study and compilation of data.

Funding for the study and editorial assistance for this manuscript were provided by ThromboGenics NV. Medical writing support was provided by Saema Magre and Diane Kwiatkoski of Quintiles. ThromboGenics provided the approval for publication.

\section{Disclosure}

Dr Reichel, Keegan Skidmore, and Jake Duker are affiliated with the New England Eye Center, Tufts Medical Center, and Tufts University School of Medicine, who received funding for this study from ThromboGenics. Drs Jaffe and Schuman are employees of Duke Eye Center, who received funding for this study from ThromboGenics. Dr Jaffe has received consulting fees from Heidelberg Engineering, Alcon/Novartis, and Neurotech during the conduct of the study. Drs Sadda and Hariri are employees of Doheny Eye Centers, University of California, Los Angeles, which received funding for this study from ThromboGenics. Dr Sadda has received a grant and consulting fees from Allergan and Genentech, and consulting fees from Alcon, Inc., Novartis International AG, and Hoffman-La Roche Ltd. The authors report no other conflicts of interest in this work.

\section{References}

1. Larsson L, Osterlin S. Posterior vitreous detachment. A combined clinical and physiochemical study. Graefes Arch Clin Exp Ophthalmol. 1985; 223(2):92-95.

2. Sebag J. Anomalous posterior vitreous detachment: a unifying concept in vitreo-retinal disease. Graefes Arch Clin Exp Ophthalmol. 2004;242(8): 690-698.

3. Johnson MW. Posterior vitreous detachment: evolution and complications of its early stages. Am J Ophthalmol. 2010;149(3):371-382.

4. Johnson MW, Brucker AJ, Chang S, et al. Vitreomacular disorders: pathogenesis and treatment. Retina. 2012;32(suppl 2):S173.

5. Schneider EW, Johnson MW. Emerging nonsurgical methods for the treatment of vitreomacular adhesion: a review. Clin Ophthalmol. 2011; 5:1151-1165.

6. Jaffe NS. Vitreous traction at the posterior pole of the fundus due to alterations in the vitreous posterior. Trans Am Acad Ophthalmol Otolaryngol. 1967;71(4):642-652.

7. Steel DH, Lotery AJ. Idiopathic vitreomacular traction and macular hole: a comprehensive review of pathophysiology, diagnosis, and treatment. Eye (Lond). 2013;27(suppl 1):S1-S21.

8. Roldan M, Serrano JM. Macular edema and vitreous detachment. Ann Ophthalmol. 1989;21(4):141-148.

9. Nasrallah FP, Jalkh AE, Van CF, et al. The role of the vitreous in diabetic macular edema. Ophthalmology. 1988;95(10):1335-1339.

10. Robison CD, Krebs I, Binder S, et al. Vitreomacular adhesion in active and end-stage age-related macular degeneration. Am J Ophthalmol. 2009; 148(1):79-82. 
11. Jackson TL, Nicod E, Simpson A, Angelis A, Grimaccia F, Kanavos P. Symptomatic vitreomacular adhesion. Retina. 2013;33(8):1503-1511.

12. Jackson TL, Nicod E, Angelis A, et al. Vitreous attachment in agerelated macular degeneration, diabetic macular edema, and retinal vein occlusion: a systematic review and metaanalysis. Retina. 2013;33: 1099-1108.

13. Girach A, Pakola S. Vitreomacular interface diseases: pathophysiology, diagnosis and future treatment options. Expert Rev Ophthalmol. 2012; 7(14):311-323.

14. Do DV, Cho M, Nguyen QD, et al. Impact of optical coherence tomography on surgical decision making for epiretinal membranes and vitreomacular traction. Retina. 2007;27:552-556.

15. Dugel PU. A new focus on the vitreous and its role in retinal function. Retina Today. 2012;4:50-53.

16. Duker JS, Kaiser PK, Binder S, et al. The international vitreomacular traction study group classification of vitreomacular adhesion, traction, and macular hole. Ophthalmology. 2013;120(12):2611-2619.

17. Stalmans P, Delaey C, de Smet MD, van Dijkman E, Pakola S. Intravitreal injection of microplasmin for treatment of vitreomacular adhesion: results of a prospective, randomized, sham-controlled phase II trial (the MIVI-IIT trial). Retina. 2010;30(7):1122-1127.
18. Benz MS, Packo KH, Gonzalez V, et al. A placebo-controlled trial of microplasmin intravitreous injection to facilitate posterior vitreous detachment before vitrectomy. Ophthalmology. 2010;117(4): 791-797.

19. de Smet MD, Gandorfer A, Stalmans P, et al. Microplasmin intravitreal administration in patients with vitreomacular traction scheduled for vitrectomy: the MIVI I trial. Ophthalmology. 2009;116(7):1349-1355.

20. Gandorfer A, Rohleder M, Sethi C, et al. Posterior vitreous detachment induced by microplasmin. Invest Ophthalmol Vis Sci. 2004;45(2): 641-647.

21. JETREA [package insert]. Iselin, NJ: ThromboGenics, Inc.; 2014.

22. Meuer SM, Myers CE, Klein BEK, et al. The epidemiology of vitreoretinal interface abnormalities as detected by spectral-domain optical coherence tomography. The Beaver Dam Eye Study. Ophthalmology. 2015;122:787-795.
Clinical Ophthalmology

\section{Publish your work in this journal}

Clinical Ophthalmology is an international, peer-reviewed journal covering all subspecialties within ophthalmology. Key topics include: Optometry; Visual science; Pharmacology and drug therapy in eye diseases; Basic Sciences; Primary and Secondary eye care; Patient Safety and Quality of Care Improvements. This journal is indexed on

Submit your manuscript here: http://www.dovepress.com/clinical-ophthalmology-journal

\section{Dovepress}

PubMed Central and CAS, and is the official journal of The Society of Clinical Ophthalmology (SCO). The manuscript management system is completely online and includes a very quick and fair peer-review system, which is all easy to use. Visit http://www.dovepress.com/ testimonials.php to read real quotes from published authors. 